\title{
Une mise au point à propos de l'article sur les Jésuites de W. Mettler
}

\begin{abstract}
L' article de W. Mettler nous intéresse pour deux raisons. Premièrement, parce que la liaison entre religion et le vote en faveur de la levée des articles sur les Jésuites et les Couvents a fait l'objet de nombreuses études (1). Secondement, l'interprétation de W. Mettler nous semble fallacieuse.
\end{abstract}

Nous ne nous attarderons pas sur la première partie de son exposé où l'auteur analyse la répartition des voix affirmatives entre cantons, bien que les conclusions qu'il tire déjà sur la base d'une simple comparaison entre pourcentages plus ou moins élevés soient extrêmement problématiques. Nous nous contenterons de relever l'affirmation selon laquelle la constance des institutions religieuses aurait déterminé dans des cantons aussi disparates que Fribourg, Zug et le Tessin le vote favorable aux Jésuites. Si cela était vrai, on comprendrait mal le faible pourcentage de oui dans le canton de Glaris ou en fait de Couvents celui-ci ne se trouve pas en mauvaise posture.

C'est toutefois sur la méthodologie que nous devons attirer l'attention des spécialistes.

Si d'une part le discours sur les couvents peut être aisément renversé par l'hypothèse non moins plausible selon laquelle les couvents pourraient avoir de quoi effrayer même des catholiques, la liaison entre religion et le vote "Jésuites" a été établie de manière mécanique et sans aucune connaissance des modéles sousjacents à une régression simple.

L'auteur ne semble pas non plus être partculièrement soucieux de la signification réelle d'un coefficient de corrélation linéaire simple. Dans l'article en cause, il semble implicitement interpréter le coefficient obtenu comme la preuve selon laquelle les protestants auraient tous voté négativement, les catholiques tous positivement. A part le fait qu'une telle conclusion serait en contradiction avec ce que l'auteur affirme en début d'analyse, à savoir l'opinion prépondérante selon laquelle les articles seraient désormais dépassés et l'espoir que la décision populaire facilite l'entente interconfessionnelle, l'interprétation donnée est erronnée.

Un coefficient de corrélation mesure l'intensité d'une relation entre 'deux variables. Le carré du coéfficient de corrélation indique la proportion de la variance de $\mathrm{Y}$ expliquée par X. En d'autres termes, $Y$ explique $96 \%$ de la variance de $Y$, si le terme de 0,959 est effectivement un coefficient de détermination $\left(r^{2}\right)$. Sur la fig. 4 , la droite de régression est ajustée au nuage des points qui représentent les cantons. Les écarts par rapport à cette droite sont donc précisément inexpliqués.

L'auteur de l'article ignore en outre qu'un coefficient de dépendance n'est identifiable en termes d'analyse causale que si l'hypothèse de la linéarité et de la non-corrélation entre facteurs implicites peuvent être considérés comme valides. La corrélation entre ces derniers et la variable explicite est supposée être quasiment nulle (2).

Un autre aspect est d'autre part complètement passé sous silence. Les estimations des coefficients de régression selon l'équation présentée:

$$
\begin{array}{ll}
Y=A_{0}+A_{1} \cdot X & \text { ou } \\
Y=\hat{a}_{0}+\hat{a}_{1} \cdot X & \text { sont } \hat{a}_{0} \text { et } \hat{a}_{1} .
\end{array}
$$

Si l'on en croit Goodmann (3), l'estimation $\hat{a}_{1}$ est égale à la proportion de $\mathrm{X}$ qui se trouve aussi en $Y$. Autrement dit, la part des catholiques votant oui; $\hat{a}_{o}$ est donc la part des noncatholiques votant de la même façon. De la sorte on obtient selon

$$
\begin{aligned}
& \mathrm{Y}=12,08+0,82 \mathrm{X} \\
& \text { ou plus exactement } \\
& \mathrm{Y}=0,12+0,82 \mathrm{X} \\
& \text { si on exprime } \mathrm{X}, \mathrm{Y} \text { par }
\end{aligned}
$$

catholiques population résidante

respectivement $\frac{\text { Oui }}{b}$, b étant le total des bulletins valables.

Ainsi, $0,12+0,82=0,94$, c'est-à-dire $94 \%$ des catholiques se sont exprimés favorablement.

Dans la communication que nous avons présentée au Congrès de sociologie de 1973 à Genève, nous avons jugé adéquat, à propos de cette votation, de prendre en considération la population résidante suisse en guise de population votante et l'attitude des protestants, parce qu'il pouvait paraftre plus intéressant d'en connaftre l'évolution que celle des catholiques. Car il était bien évident que ces derniers auraient voté oui.

A l'aide d'un modèle linéaire, que nous avons préalablement essayé de fonder sur la base de 
l'unanimité des consignes de l'Eglise, de l'Etat et des partis politiques, nous avons obtenu le tableau suivant (4):

Population résidante suisse

selon l'appartenance religieuse (1970)

\begin{tabular}{lcccc} 
& $\begin{array}{c}\text { ont voté } \\
\text { oui }\end{array}$ & & \multicolumn{2}{c}{$\begin{array}{c}\text { ont voté } \\
\text { non }\end{array}$} \\
\cline { 2 - 2 } Protestants & $24 \%$ & & $76 \%$ & $100 \%$ \\
Non-protestants & $94 \%$ & & $6 \%$ & $100 \%$
\end{tabular}

A la lumière de ces résultats, la justification que W. Mettler cherche à attribuer à sa méthode est dépourvue de sens. Le clivage qu'il semble y voir n'est pas aussi profond. In mérite un jugement plus nuancé. Finalement, nous ne voyons pas quelles pourraient être les conséquences de cette votation en matière de paysage. S'il craint une ingérence des couvents dans le paysage, son appréhension ne peut se dire fondée. En tous cas, sa régression ne le permet pas.
(1) NZZ, "Die konfessionelle Struktur des Abstimmungsergebnisses", Differenzierte Stellungnahme der Protestanten zu den Ausnahmeartikeln, 25 mai $1973, \mathrm{Nr} .239$, p. 24 Basler Nachrichten, War es wirklich nur die Konfession? 2 juin 1973, Nr. 127, p. 3/4 Gilg (Peter), Das reformierte Nein vom 20. Mai 1973, Reformatio, 22 (9), pp. 455466.

(2) Boudon (Raymond), l'analyse mathématique des faits sociaux, Paris, 1970 , p. 98.

(3) Goodman (Leo A.), Some alternatives to ecological correlations, American Journ. of Sociology, 64, 1959, pp. 610-625.

(4) Wyss (Marc), un aspect de la résistence au changement en Suisse: l'affaire des Jésuites (communication au 2ème congrès de la Société Suisse de Sociologie, 1973, rapport multicopié), p. 4.

Marc Wyß, lic. rer. pol., Institut für Orts-, Regional- und Landesplanung, Weinbergstraße 35, 8006 Zürich

\section{Literaturbesprechung}

AUBERT H. J. und MUELLER U. E. , Sri LankaCeylon, Kümmerly + Frey, Bern, 1974, Fr.58. Ceylon, das jüngst mit Sri Lanka seinen vor 2000 Jahren schon in altindischen Epen erwähnten Namen wieder offiziell angenommen hat, vereinigt eine unendliche Vielfalt in seinen Landschaftsformen und Kulturen, Rassen und Religionen. Der vorliegende Bildband trägt vor allem dieser Mannigfaltigkeit Rechnung. In seinen prächtigen, grösstenteils farbigen Aufnahmen bringt er die landschaftliche Spannung von tropischem Regenwald bis zu wüstenhaften Dünengebieten, von flachen Küstenräumen zu den Gebirgsregionen voll zur Geltung. Ebenso spricht die schillernde Vielfalt der Wirtschaftsund Lebensformen aus den Bildern, beispielsweise Brandrodungskultur, Reisanbau auf sorgfältig angelegten Terrassen, selbstversorgende Gartenkulturen und marktorientierte Plantagen- wirtschaft (vgl. Farbbeilage in Heft 1/1974 der Geographica Helvetica). Eingehende Berücksichtigung der geschichtlichen Entwicklung, des Ausdrucks von kulturellen und religiösen Eigenarten und deren Grundlagen sowie des gegenwärtigen Wandels in seiner vollen Problematik rundet Bild und Beschreibung zur ansprechenden Länderkunde dieser "Perle des Indischen Ozeans" ab. Im Text werden geographische Sachverhalte ausführlich dargelegt und durch Einzelbeiträge namhafter Kenner der Insel über Gestalt und Aufbau, Vegetation und den Buddhismus in wertvoller Weise ergänzt. Das Werk vermittelt alle wesentlichen Informationen über das allgemein wenig bekannte Sri Lanka, das schon seit einiger Zeit auch in den Bereich des internationalen Tourismus einbezogen wurde. 